\title{
NIVELES DE CARNITINA EN PACIENTES QUE RECIBEN NUTRICIÓN PARENTERAL TOTAL
}

\author{
Carnitine levels in patients receiving total parenteral nutrition \\ Lizbeth Cajaleón $\mathrm{F}^{1}$, Rocío Páucar B ${ }^{1}$, María Ocaña $\mathrm{P}^{2}$, Elena Benavides $\mathrm{R}^{1}$. \\ ${ }^{1}$ Facultad de Farmacia y Bioquímica, Universidad Nacional Mayor de San Marcos, ${ }^{2}$ Hospital Nacional Edgardo Rebagliati \\ Martins
}

\section{RESUMEN}

El aporte de lípidos en la Nutrición Parenteral Total (NPT) viene administrándose mediante ácidos grasos de cadena larga en los pacientes del Hospital Edgardo Rebagliati Martins. En el presente estudio se compararon los niveles plasmáticos de carnitina de pacientes que reciben NPT basada en triacilglicéridos de cadena larga (TCL) y los que reciben NPT basada en TCL/TCM (triacilglicéridos de cadena media) por tiempo prolongado ( $\geq 7$ días). Previamente se determinaron los niveles plasmáticos de carnitina en personas aparentemente sanas, en pacientes con desnutrición severa y pacientes que recibieron NPT por más de 20 días. Al final del estudio se hallaron los siguientes valores medios de carnitina plasmática, expresadas en $\mu \mathrm{mol} / \mathrm{L}$ : personas aparentemente sanas 34,26, que se halla dentro de los valores normales; en pacientes con desnutrición severa, 68,29; pacientes con NPT por más de 20 días 23,64, pacientes con NPT basada en TCL ( $\geq 7$ días) 43,27; pacientes con NPT basada en TCL/TCM ( $\geq 7$ días) 26,68; hallándose sólo diferencia significativa al comparar los grupos que reciben NPT basada TCL y los que reciben NPT basada en TCL/TCM ( $\geq 7$ días), $p<0.05$.

Palabras clave: Carnitina, ácidos grasos, nutrición parenteral total, lípidos.

\section{SUMMARY}

The contribution of lipids in the Total Parental Nutrition (TPN) is given by for long chain fatty acids (LCT) in patients Edgardo Rebagliati Martins Hospital. In the present study compared the plasma levels of carnitine in patients receiving TPN based on long chain and medium chain triacilglicéridos (LCT/MCT) for prolonged time ( $\geq 7$ days). Previously identified carnitine plasma levels in apparently healthy persons and patients with severe hunger and those who receive TPN for more than 20 days. At the end of the study found the following average values of plasma carnitine, expressed in umol / L: 34, 26 for apparently healthy people who are within the normal range, in patients with severe malnutrition ( $\geq 7$ days) 43,27 ; TPN patients based on LCT / TCM ( $\geq 7$ days) 26,68 . We found only significant difference when comparing groups based on TPN receiving LCT and TPN-based TCM / TCL ( $\geq 7$ days), $p<0.05$.

Keywords: Carnitine, total parental nutrition, lipids.

\section{INTRODUCCIÓN}

os pacientes sometidos al estrés hipermetabólico y desnutrición presentan un estado metabólico alterado en comparación con sujetos normales ${ }^{(1)}$. El aporte energético viene dado por los nutrientes que nos brindan los alimentos. Los pacientes que no pueden recibir este aporte por la dieta y digestión tradicional, cubren sus requerimientos gracias al Soporte Nutricional Especial, en el que una de las principales fuentes de energía proviene de los lípidos ${ }^{(2)}$. Es importante el buen aprovechamiento de este nutriente.

Los ácidos grasos libres deben ser transportados al interior de la mitocondria para su posterior $\beta$-oxidación y con lo cual se logrará el aporte energético ${ }^{(2)}$.

La carnitina es una molécula esencial para el transporte de ácidos grasos de cadena larga al interior de la membrana mitocondrial ${ }^{(3)}$. Es sintetizada a partir de lisina y metionina en el hígado y el riñón (4). La carnitina libre en plasma varía según el sexo, la raza y principalmente el estado de salud de la persona ${ }^{(3)}$, siendo los valores plasmáticos normales de 30-50 $\mu \mathrm{mol} / \mathrm{L}$ y deficiente si está por debajo de $20 \mu \mathrm{mol} / \mathrm{L}^{(5)}$.

El aporte de lípidos en la Nutrición Parenteral Total (NPT) viene dándose principalmente por ácidos grasos de cadena larga. Sin embargo, estos lípidos son asociados con infiltración de grasa a los tejidos, bajo aclaramiento de la circulación, hipertrigliceridemia, deterioro de la función inmunitaria e interferencia en 
el sistema retículoendotelial ${ }^{(6)}$.

Se considera que, las nuevas emulsiones que contienen ácidos grasos de cadena mediana son una mejor fuente de energía, ya que son removidos rápidamente de la circulación, oxidados eficientemente y su almacenamiento en tejido adiposo e hígado es limitado ${ }^{(6)}$. Estos llegan a ser la fuente de energía de pacientes en estado crítico hipermetabólico.

Los ácidos grasos de cadena mediana pueden ingresar a la mitocondria para su posterior $\beta$-oxidación, independientemente del sistema de transporte mediado por la carnitina ${ }^{(7)}$.

Usualmente, los pacientes quirúrgicos con NPT no reciben carnitina ${ }^{(8)}$ debido a que el estrés quirúrgico incrementa la oxidación de ácidos grasos ${ }^{(9,10)}$. Numerosos estudios plantean mejorar el aporte energético de la NPT con la adición de suplementos de carnitina, especialmente en aquellas cuya fuente energética de lípidos la constituyen los ácidos grasos de cadena larga.

En el Perú no existen datos respecto a niveles de carnitina que permitan estimar valores normales y de deficiencia para nuestra población, ni sobre la importancia del uso de un determinado tipo de emulsión basada en TCL o una mixtura de TCL/TCM que nos pueda dar luces sobre la mejor fuente de energía para estos pacientes.

\section{METODOLOGÍA}

El estudio experimental se realizó entre los meses de enero y junio del año 2007 en el Hospital Edgardo Rebagliati Martins ubicado geográficamente en el distrito de Jesús María, Lima - Perú.

Se realizó previamente un estudio de campo para estimar los valores reales en nuestra población, formándose tres grupos:

- Personas aparentemente sanas: 10

- Pacientes con desnutrición severa que no reciben NPT: 04

- Pacientes que reciben NPT $\geq 20$ días : 06

Luego del estudio de campo se procedió con el análisis de los grupos de la población en estudio:

- Pacientes adultos con NPT basada en ácidos grasos de cadena larga (TCL) por tiempo prolongado $(\geq 7$ días): 10

- Pacientes adultos con NPT basada en ácidos grasos de cadena larga y cadena mediana (TCL/TCM) por tiempo prolongado ( $\geq 7$ días): 08
La toma de muestra de sangre fue realizada por el personal de enfermería de la Unidad de Soporte Nutricional Artificial (USNA).

Días de toma de muestra de sangre:

- En el día 0 (día antes de recibir la Nutrición Parenteral Total).

- En el día 7 (séptimo día de administración de la Nutrición Parenteral Total).

\section{Determinación de carnitina}

Las concentraciones fueron determinadas en plasma mediante el método enzimático espectrofotométrico. El ensayo se basa en la reacción entre la L-carnitina y acetil-CoA en presencia de la enzima carnitina aciltransferasa (CAT). La coenzima A (CoA-SH) obtenida reacciona con el reactivo 5-5' di-tiobis 2-nitrobenzoato (DTNB) formando 5-tio-2-nitrobenzoato $\left(\mathrm{TNB}^{-}\right)$el cual absorbe la luz a 412 ๆm.

L-carnitina $+A c \mathrm{Co} A \stackrel{\mathrm{CAT}}{\longrightarrow}$ Acetil $-L-$ carnitina $+\mathrm{Co} A-S H$
$\mathrm{Co} A-\mathrm{SH}+\mathrm{DTNB} \longrightarrow \mathrm{Co} A-\mathrm{S}-\mathrm{NB}+\mathrm{TNB}^{-}+\mathrm{H}^{+}$

\section{RESULTADOS Y DISCUSIÓN}

En la figura 1 se muestran los valores promedio de carnitina obtenidos en el trabajo de campo; asimismo, la tabla 1 presenta la comparación de medias por grupos.

El grupo de personas aparentemente sanas, tiene una media de 34,26 $\mu \mathrm{mol} / \mathrm{L}$, este valor se encuentra dentro del rango de los valores normales establecidos internacionalmente (Sigma Tau Pharmaceutical) ${ }^{(5)}$.

Los pacientes con desnutrición severa no tenían una fuente exógena de combustible pues no recibían ningún tipo de alimentación. Según Steiber y Cecil ${ }^{(11,12)}$; aproximadamente el 60 a $75 \%$ de carnitina total proviene de alimentos que contienen carnitina, lisina y metionina, por lo que se podría esperar una disminución en los niveles plasmáticos de carnitina en este grupo de pacientes; sin embargo presentaron valores por encima de los normales $(68,29 \mu \mathrm{mol} / \mathrm{L})$.

Khan ${ }^{(13)}$ reporta bajos niveles plasmáticos de carnitina en pacientes con desnutrición proteico-calórica; esto contradice los resultados obtenidos, posiblemente por el número limitado de pacientes para este grupo (4 sujetos) y la selección en un número limitado de parámetros bioquímicos.

El grupo de pacientes con NPT por más de 20 
Tabla 1. Comparación de medias por grupos.

\begin{tabular}{lcc}
\hline \multicolumn{1}{c}{ Grupos } & $\mathbf{n}$ & $\begin{array}{c}\text { Media } \\
(\mu \mathbf{m o l} / \mathbf{L})\end{array}$ \\
\hline Aparentemente sanos & 10 & 34,26 \\
Desnutrición Severa & 4 & 68,29 \\
NPT $\geq 20$ días & 6 & 23,64 \\
\hline
\end{tabular}

días, tiene una media de $23,64 \mu \mathrm{mol} / \mathrm{L}$, este valor se encuentra por debajo del rango de los valores normales. $\mathrm{Al}$ respecto Bowyer et al. ${ }^{(4)}$, reportan la deficiencia de carnitina en adultos que reciben NPT por más de 20 días, lo que se confirma con nuestros resultados.

\section{Comparación entre medias del esta- do basal y primera toma de muestra}

En la figura 2 se presentan los valores promedio de carnitina basal y luego de la $1^{\text {ra }}$ toma de muestra para el grupo que recibe NPT basada en TCL $(n=10)$. La diferencia entre las medias no es significativa $(p=$ 0.996 )

La figura 3 presenta los valores de carnitina basal y luego de la $1^{\text {ra }}$ toma de muestra para el grupo que recibe NPT basada en TCL/TCM $(n=8)$. La diferencia entre las medias del basal y ${ }^{\text {ra }}$ toma de muestra no es significativa para $\alpha=0,05(p=0,069)$; pero sí es significativa para $\alpha=0,1$.

\section{Comparación de las medias de la primera toma de muestra entre NPT basada en TCL y NPT basada en TCL/ TCM}

Luego de 7 días de NPT con los lípidos respectivos se encontraron: en el caso de pacientes con NPT basada en TCL, valores plasmáticos de carnitina por encima de los hallados en los pacientes con NPT basada en TCL/TCM, 43,27 $\mu \mathrm{mol} / \mathrm{L}$ y 26,68 $\mu \mathrm{mol} / \mathrm{L}$ respectivamente; la diferencia se podría atribuir a que los lípidos de cadena larga necesitan de carnitina como lanzadera para su $\beta$-oxidación y por lo tanto ésta será transportada del área de almacenamiento o síntesis por medio del torrente sanguíneo al área de necesidad metabólica. En el caso de la mezcla se necesita de este transportador en menor cantidad porque contiene un 50\% de ácidos grasos de cadena media, los cuales ingresan a la mitocondria de manera independiente del sistema de transporte de carnitina, utilizando sus reservas en menor cantidad.

Las varianzas son homogéneas. Aplicando la prueba t de Student se halla una diferencia estadísticamente significativa, $p<0$, 05. Por tanto, se concluye que la diferencia de medias observada es significativa $(\alpha=0,05)$

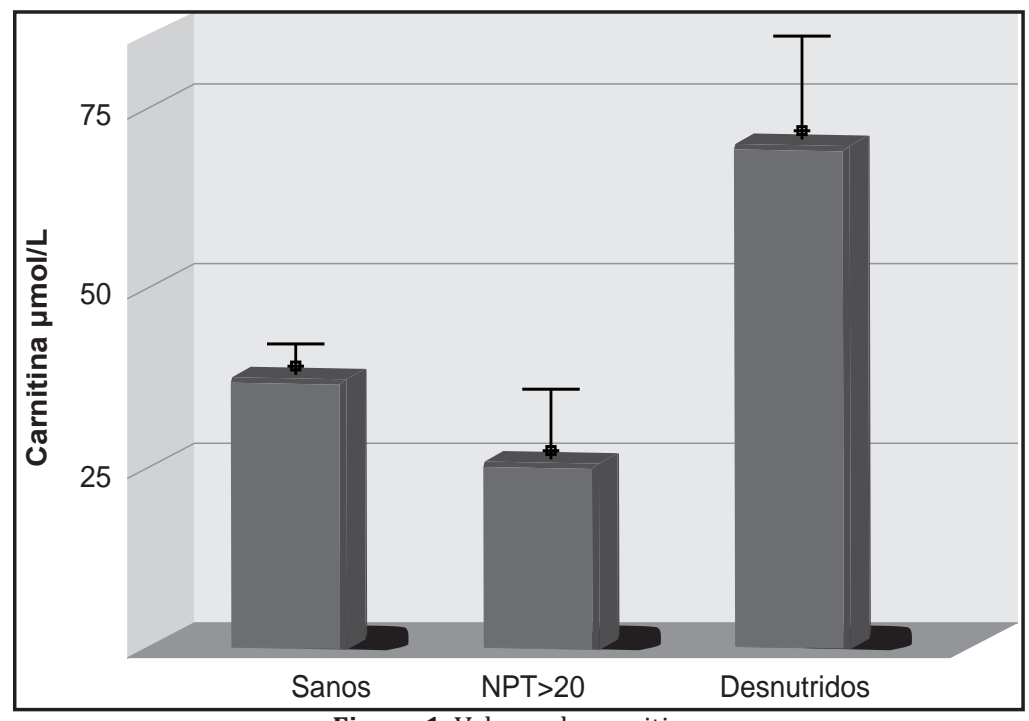

Figura 1. Valores de carnitina.

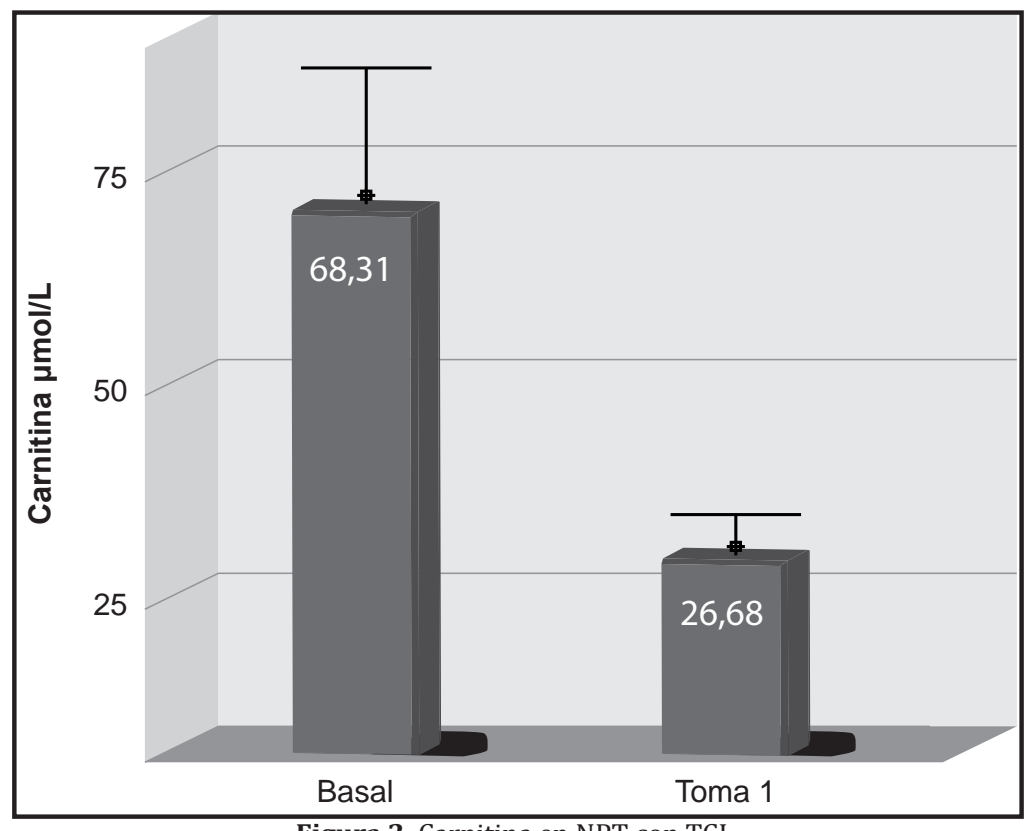

Figura 2. Carnitina en NPT con TCL. 


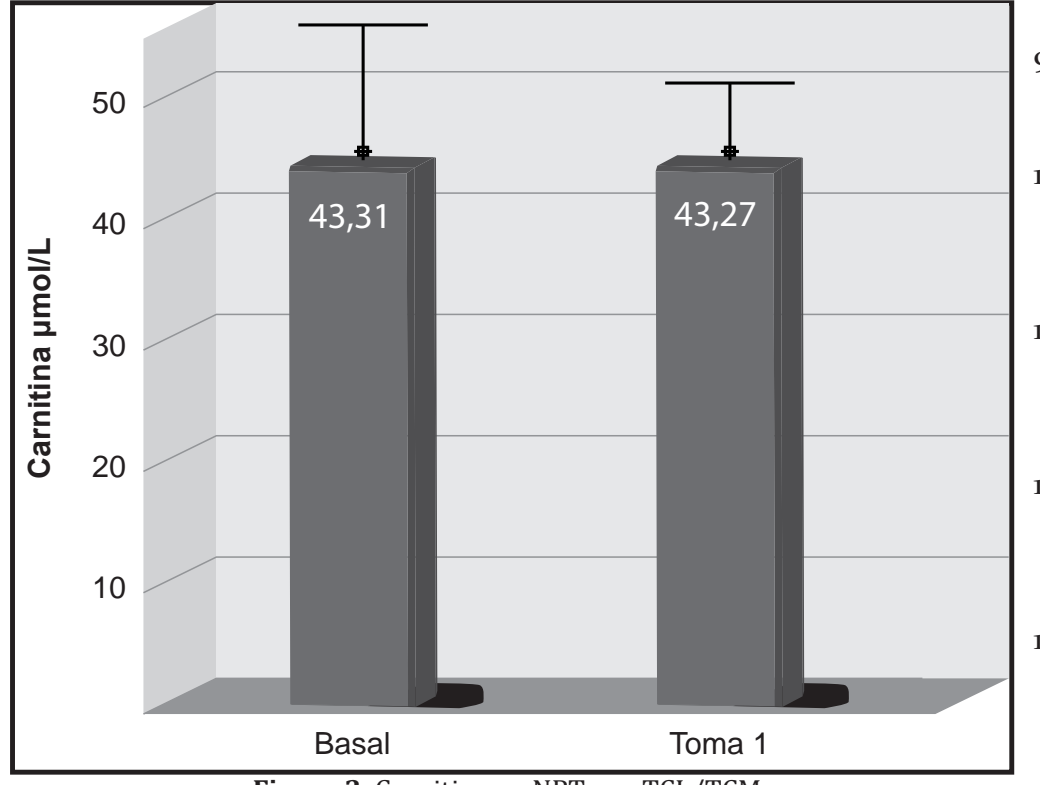

Figura 3. Carnitina en NPT con TCL/TCM.

\section{CONCLUSIÓN}

Se han hallado mayores niveles plasmáticos de carnitina en el grupo de pacientes que reciben NPT basada en TCL que en los que reciben NPT basada en TCL/TCM.

\section{REFERENCIAS BIBLIOGRÁFICAS}

1. Tucker H. Fundamentos de la ayuda nutricional en el paciente hipermetabólico: conocimientos actuales y perspectivas. Nutrición Clínica 2003: 85-99.

2. Mora R J. Soporte nutricional especial. Editorial Médica Panamericana. Bogota, 2002: 251.

3. Bremer J. Carnitine-metabolism and functions. Physiol Rev 1983; 63:1420-1468.

4. Bowyer BA, Fleming CR, Ilstrup D et al. Plasma carnitine levels in patients receiving home parenteral nutrition. Am J Clin Nutr 1986;43: 85-91.

5. Sigma Tau Pharmaceutical Inc. Carnitor ${ }^{\circledR}$ levocarnitine. Gaithersburg. MD 20877.

6. Nijveldt RJ, Tan AM, Prins HA, De Jong D. Use of a mixture of medium-chain triglycerides and long-chain triglycerides versus long-chain triglycerides in critically surgical patients: a randomized prospective double blind study. Clinical Nutrition 1998; 17: 23-29.

7. Hahn P, Allardyce DB, Frohlich J. Plasma carnitine levels during total parenteral nutrition of adult surgical patients. Am J Gin Nutr 1982; 36: 569-572.

8. Pichard R, Schutz R, Chiolero T, Schindler Z, Furst J. Clinical relevance of L-carnitina suplemented total parenteral nutrition in post operative trauma. Metabolic effects of continuous or acute carnitine administration with special reference to fat oxidation and nitrogen utili- zation. Am J Clin Nutr 1989; 49:283-289.

9. Hill GL, Church J. Energy and protein requirements of general surgical patients requiring intravenous nutrition. Br J Surg 1984; 71: 1-9.

10. Nordenström J, Carpentier YA, Askanazi J et al. Metabolic utilization of intravenous fat emulsion during total parenteral nutrition. Ann Surg 1982; 196: 221-231.

11. Steiber K, Hoppel. Carnitine: a nutritional, biosynthetic, and funtional perspective. Molecular Aspects of Medicine 2004; 25: 455473 .

12. Cecil J. Nutritional Supplements: Amino acids and their derivatives. American Journal of Pharmaceutical Education 2003; 66: 159161.

13. Khan Land Bamji MS. Plasma carnitine levels in children with protein calorie malnutrition before and after rehabilitation. Clin Chim Acta 1977; 75:163-166.
Manuscrito recibido el: 30/11/2009

Aceptado para su publicación el: 23/02/2010

\section{Correspondencia:}

Nombre: Elena Rafaela Benavides Rivera Dirección: Jr. Apurímac № 3672, Urb. Perú - Lima 31. e-mail: elenabenavidesr@gmail.com 